TRANSACTIONS OF THE

AMERICAN MATHEMATICAL SOCIETY

Volume 362, Number 10, October 2010, Pages 5169-5187

S 0002-9947(2010)04831-7

Article electronically published on May 19, 2010

\title{
A HILBERT-MUMFORD CRITERION FOR POLYSTABILITY IN KAEHLER GEOMETRY
}

\author{
I. MUNDET I RIERA
}

\begin{abstract}
Consider a Hamiltonian action of a compact Lie group $K$ on a Kaehler manifold $X$ with moment map $\mu: X \rightarrow \mathfrak{k}^{*}$. Assume that the action of $K$ extends to a holomorphic action of the complexification $G$ of $K$. We characterize which $G$-orbits in $X$ intersect $\mu^{-1}(0)$ in terms of the maximal weights $\lim _{t \rightarrow \infty}\left\langle\mu\left(e^{\mathbf{i} t s} \cdot x\right), s\right\rangle$, where $s \in \mathfrak{k}$. We do not impose any a priori restriction on the stabilizer of $x$. Under some mild restrictions on the action $K \circlearrowright X$, we view the maximal weights as defining a collection of maps: for each $x \in X$,

$$
\lambda_{x}: \partial_{\infty}(K \backslash G) \rightarrow \mathbb{R} \cup\{\infty\},
$$

where $\partial_{\infty}(K \backslash G)$ is the boundary at infinity of the symmetric space $K \backslash G$. We prove that $G \cdot x \cap \mu^{-1}(0) \neq \emptyset$ if: (1) $\lambda_{x}$ is everywhere nonnegative, (2) any boundary point $y$ such that $\lambda_{x}(y)=0$ can be connected with a geodesic in $K \backslash G$ to another boundary point $y^{\prime}$ satisfying $\lambda_{x}\left(y^{\prime}\right)=0$. We also prove that the maximal weight functions are $G$-equivariant: for any $g \in G$ and any $y \in \partial_{\infty}(K \backslash G)$ we have $\lambda_{g \cdot x}(y)=\lambda_{x}(y \cdot g)$.
\end{abstract}

\section{INTRODUCTION}

Let $K$ be a compact connected Lie group with Lie algebra $\mathfrak{k}$, let $X$ be a (not necessarily compact) Kaehler manifold, and assume that there is an action $K \circlearrowright$ $X$ by holomorphic isometries (in particular, preserving the symplectic form) and admitting a moment map $\mu: X \rightarrow \mathfrak{k}^{*}$. Since $\mu$ is by definition $K$-equivariant, the action of $K$ on $X$ preserves the zero level set $\mu^{-1}(0)$ and the quotient $\mu^{-1}(0) / K$ carries a natural structure of a stratified symplectic manifold; see [SL]. Let $G$ be the complexification of $K$, and assume that the action of $K$ extends to a holomorphic action of $G$, so that the map $G \times X \ni(g, x) \mapsto g \cdot x \in X$ is holomorphic. If $X$ is compact, the action of $K$, which by assumption preserves the complex structure on $X$, extends automatically (this follows essentially from a theorem of Bochner and Montgomery $\mathrm{BM}$; see also GS]). If $X$ is not compact, then such an extension need not exist (as an example, take the group of rotations of the open unit disc in $\mathbb{C})$.

A very natural and important question, which has been extensively treated in the literature, is to find a good notion of a quotient of $X$ by the action of $G$, carrying a structure of a (possibly singular) Kaehler manifold induced in some way from the structure in $X$. The most naive possibility, taking the space of orbits $X / G$ with the quotient topology, will not even be Hausdorff in general, so there is no hope to

Received by the editors April 4, 2008 and, in revised form, May 20, 2008.

2010 Mathematics Subject Classification. Primary 53D20; Secondary 32M05.

Key words and phrases. Hamiltonian actions, Kaehler geometry, Hilbert-Mumford criterion.

(C)2010 American Mathematical Society 
provide it with a structure of a singular Kaehler manifold. To avoid this pathology one can restrict the attention to a big $G$-invariant subset $X^{*} \subset X$, obtained after removing some bad $G$-orbits in $X$, such that the quotient space $X^{*} / G$ is Hausdorff. This faces us with the problem of defining $X^{*}$ in a natural way, satisfying the previous condition and at the same time being as big as possible (for example, we would like $X^{*}$ to be dense in $X$ ).

A systematic way of defining $X^{*}$ uses the moment map of the action of $K$. (For the results stated in this paragraph and the next one, see the survey $\mathrm{HH}$ ] and the references therein.) One says that $x \in X$ is semistable if the closure of $G \cdot x$ intersects $\mu^{-1}(0)$. Then the set $X^{s s} \subset X$ of semistable points is open and the relation which identifies two orbits in $X^{s s}$ if their closures intersect is an equivalence relation. The quotient space $X / / G$ of this equivalence relation carries a natural structure of a (possibly singular) holomorphic space, and the projection $X^{s s} \rightarrow X / / G$ is holomorphic. On the other hand, if one defines $X^{p s} \subset X$ as the set of points whose $G$-orbit intersects $\mu^{-1}(0)$ (we call such points polystable), then the inclusion $X^{p s} \subset X^{s s}$ induces a homeomorphism $X^{p s} / G \simeq X / / G$, so that one can take $X^{p s}$ as a good choice for $X^{*}$. This motivates the following question.

Question 1.1. Which $G$-orbits $\mathcal{O}=G \cdot x \subset X$ intersect $\mu^{-1}(0)$ ? If $\mathcal{O}$ is such an orbit, how many $K$-orbits does $\mathcal{O} \cap \mu^{-1}(0)$ contain?

In the first question we would like some characterization of the points $x \in X$ such that $G \cdot x \cap \mu^{-1}(0) \neq \emptyset$ in terms of the symplectic geometry of the action $K \circlearrowright X$. The answer to the second question (namely, that $\mathcal{O} \cap \mu^{-1}(0)$ contains at most one $K$-orbit) is well known and follows from an easy argument, which we recall in Section 4.1 below. A refinement of this is the statement that $X / / G$ is homeomorphic to the symplectic quotient $\mu^{-1}(0) / K$, and that the holomorphic structure on $X / / G$ defined in $[\mathrm{HH}$ is compatible with it, in the sense that they combine to define a structure of a stratified Kaehler manifold (see $[\mathrm{HH}, \mathbf{S}$ ).

A partial characterization of the $G$-orbits intersecting $\mu^{-1}(0)$ was given in $\mathrm{M}$ ] in terms of the maximal weights $\lambda(x ; s)$, defined for any $s \in \mathfrak{k}$ to be

$$
\lambda(x ; s)=\lim _{t \rightarrow \infty}\left\langle\mu\left(e^{\mathbf{i} t s} \cdot x\right), s\right\rangle \in \mathbb{R} \cup\{\infty\}
$$

(this limit exists; see Section 3.2 below). A point $x \in X$ is defined in $[\mathrm{M}$ to be analytically stable if $\lambda(x ; s)>0$ for any nonzero $s \in \mathfrak{k}$, and [M, Theorem 5.4] states that $x$ is analytically stable if and only if $G \cdot x \cap \mu^{-1}(0) \neq \emptyset$ and the stabilizer $G_{x}$ is finite. Apart from the restriction to points with finite stabilizers, the point of view in $[\mathrm{M}]$ has the inconvenience that it does not seem to allow any straightforward proof that if $x$ is analytically stable, then $g \cdot x$ is also analytically stable for any $g \in G$. Roughly speaking, this is proved in $\underline{\mathrm{M}}$ as a consequence of the characterization of analytic stability in terms of the so-called linear properness of the integral of the moment map, which essentially amounts to $\mathrm{M}$, Theorem 5.4]. (The equivariance property of the moment map allows one to prove that $\lambda(k \cdot x ; s)=\lambda(x ; \operatorname{Ad}(k)(s))$ for any $k \in K$, which clearly implies that analytic stability is a property of $K$-orbits, but there is no obvious action of $G$ on $\mathfrak{k}$ extending the adjoint action of $K$ giving a similar $G$-equivariance property of the maximal weights.)

It is easy to deduce from the results in $[\mathrm{M}]$ that if $\lambda(x ; s)<0$ for some $s$, then $G \cdot x \cap \mu^{-1}(0)=\emptyset$. Hence if $G \cdot x \cap \mu^{-1}(0) \neq \emptyset$, then $\lambda(x ; s) \geq 0$ for any $s$. But this is not a sufficient condition: to decide whether $G \cdot x \cap \mu^{-1}(0)$ is nonempty one needs to control in some way which of the maximal weights vanish. 
A well-known and elementary example which illustrates these phenomena is given by the action of the isometries of $S^{2}$ on tuples of points. Here $S^{2}$ denotes the unit sphere in $\mathbb{R}^{3}$ centered at 0 and endowed with the round metric. Let $K=\mathrm{SO}(3, \mathbb{R})$ be the group of orientation-preserving isometries of $S^{2}$. The complexification of $K$ is $G=\operatorname{PSL}(2, \mathbb{C})$, which can be identified with the holomorphic automorphisms of $S^{2} \simeq \mathbb{C P}^{1}$. Let $X=\left(S^{2}\right)^{4}$ and take on $X$ the product Kaehler structure. The diagonal action of $K$ on $X$ clearly preserves the holomorphic structure and the symplectic form, and a moment map for it is given by sending any tuple $\left(x_{1}, \ldots, x_{4}\right) \in S^{2}$ to its center of mass $\frac{1}{4}\left(x_{1}+\cdots+x_{4}\right)$ (here we identify $\mathbb{R}^{3} \simeq \mathfrak{s o}(3, \mathbb{R})^{*}$ using the vector product in $\left.\mathbb{R}^{3}\right)$. The reader can easily check that, if $\left\{x_{1}, x_{2}, x_{3}, x_{4}\right\} \subset S^{2}$ are distinct points, then

- $x=\left(x_{1}, x_{2}, x_{3}, x_{4}\right) \in X$ is analytically stable,

- $x^{\prime}=\left(x_{1}, x_{1}, x_{2}, x_{3}\right) \in X$ satisfies $G \cdot x^{\prime} \cap \mu^{-1}(0)=\emptyset$ but all maximal weights $\lambda\left(x^{\prime} ; s\right)$ are nonnegative,

- $x^{\prime \prime}=\left(x_{1}, x_{1}, x_{2}, x_{2}\right) \in X$ satisfies $G \cdot x^{\prime \prime} \cap \mu^{-1}(0) \neq \emptyset$, but some of the maximal weights $\lambda(x ; s)$ vanish,

- $x^{\prime \prime \prime}=\left(x_{1}, x_{1}, x_{1}, x_{2}\right)$ has some negative weights.

In the usual sense in GIT, $x$ is stable, $x^{\prime}$ is semistable and the closure of the orbit $G \cdot x^{\prime}$ contains $G \cdot x^{\prime \prime}$ (and hence meets $\mu^{-1}(0)$ ), and $x^{\prime \prime \prime}$ is not semistable (we consider here the polarization of $X$ which gives the same weight to each of the four points; this corresponds to the fact that the Kaehler structure on $X$ is the product of four copies of the Kaehler structure on $S^{2}$ ).

A complete solution to Question 1.1 was given by A. Teleman in [T]. However, the result in [T] has some limitations. First, it is assumed that $X$ satisfies a condition called energy-completeness (see $T$, Definition 3.8]). Second, when giving a sufficient condition for a point $x \in X$ to satisfy $G \cdot x \cap \mu^{-1}(0)$ it is assumed that the Lie algebra $\mathfrak{g}_{x}$ of the stabilizer $G_{x}$ is reductive (see [T, Definition 3.12]). This is a little bit unsatisfactory: it might be preferable to obtain the reductivity of $\mathfrak{g}_{x}$ as a consequence of a simpler condition involving exclusively the maximal weights, not any information on the stabilizer of $x$.

In this paper we propose an alternative answer to Question 1.1 based on viewing the maximal weights as defining a function on the boundary $\partial_{\infty}(K \backslash G)$ of the symmetric space $K \backslash G$. Such a boundary exists by the general theory of Hadamard spaces, of which $K \backslash G$ is an instance (see $[\mathrm{B}, \mathrm{E}]$ ), and it is homeomorphic to a sphere of dimension one unit less than that of $K \backslash G$.

To prove our results we still require some technical restriction to be satisfied by $X$; namely, we assume that the vector fields generated by the infinitesimal action grow at most linearly with respect to the distance function from a given base point. More precisely, we take any invariant metric on $\mathfrak{k}$ and we require the following.

Assumption 1.2. The manifold $X$ is connected, and there exists a point $x_{0} \in X$ and a constant $C>0$ such that for any $x \in X$ and any $s \in \mathfrak{k}$ we have

$$
\left|\xi_{s}(x)\right| \leq C|s|\left(1+d_{X}\left(x_{0}, x\right)\right)
$$

where $\xi_{s} \in \mathfrak{C}^{\infty}(T X)$ is the vector field generated by the infinitesimal action of $s$, and $d_{X}$ denotes the geodesic distance between points of $X$. 
This condition is satisfied e.g. when $X$ is compact or when $X$ is a vector space and the action of $K$ is linear. The connectedness condition will be used in Lemma 3.3 below to derive a quadratic bound on the moment map. Remark that, unlike in [T, we do not impose any completeness condition on $X$. This of course prevents us from obtaining any characterization of semistability, which naturally involves taking the closure of orbits (see the comments at the end of the Introduction).

Using Assumption (1.2) we construct in Section 3.3 the maximal weight function

$$
\lambda_{x}: \partial_{\infty}(K \backslash G) \rightarrow \mathbb{R} \cup\{\infty\}
$$

for any $x \in X$ as an appropriate limit of a normalization of the integral of the moment map

$$
\psi_{x}: K \backslash G \rightarrow \mathbb{R} .
$$

The integral of the moment map was defined in $[\mathrm{M}$ as a function $G \rightarrow \mathbb{R}$, and it was observed in [M, Proposition 3.4] that it is invariant under the action of $K$ on the left on $G$. The boundary $\partial_{\infty}(K \backslash G)$ carries an action of $G$ extending the right action on $K \backslash G$ by isometries, and we prove in Lemma 3.6 that for any $x \in X$ and $g \in G$ we have

$$
\lambda_{g \cdot x}(y)=\lambda_{x}(y \cdot g) .
$$

This property is a consequence of the cocycle property satisfied by the integral of the moment map (see formula (3.4) below).

We say that $x \in X$ is analytically stable if for any $y \in \partial_{\infty}(K \backslash G)$ we have $\lambda_{x}(y)>$ 0 . A point $x \in X$ is said to be analytically polystable if for any $y \in \partial_{\infty}(K \backslash G)$ we have $\lambda_{x}(y) \geq 0$ and for any $y \in \partial_{\infty}(K \backslash G)$ such that $\lambda_{x}(y)=0$ there exists some $y^{\prime} \in \partial_{\infty}(K \backslash G)$ such that $\lambda_{x}\left(y^{\prime}\right)=0$ and the points $y, y^{\prime}$ can be connected by a geodesic in $K \backslash G$.

The next theorem is the main result of the paper. It will be proved in Section 4 .

Theorem 1.3. Let $x \in X$ be any point and let $G_{x}=\{g \in G \mid g \cdot x=x\}$ be its stabilizer.

(1) If $x$ is analytically stable (resp. analytically polystable), then $g \cdot x$ is analytically stable (resp. analytically polystable) for each $g \in G$.

(2) The intersection $G \cdot x \cap \mu^{-1}(0)$ consists of at most one $K$-orbit.

(3) $x$ is analytically stable if and only if $G \cdot x \cap \mu^{-1}(0) \neq \emptyset$ and $G_{x}$ is finite.

(4) $x$ is analytically polystable if and only if $G \cdot x \cap \mu^{-1}(0) \neq \emptyset$. If this is the case, then $G_{x}$ is reductive.

Note that statement (1) follows immediately from the equivariance property (1.1). (2) and (3) are well known, but we also prove them for completeness (the proof we give of (3), using the index of maps between spheres, is new to the best of our knowledge).

The second main result of the paper is a characterization of analytic polystability in terms of the weights $\lambda(x ; s)$. For any $s \in \mathfrak{k}$ of unit length, let $e_{s}$ be the boundary point of $K \backslash G$ to which the geodesic $t \mapsto\left[e^{\mathrm{i} t s}\right]$ converges as $t \rightarrow \infty$. We have: $\lambda(x ; s)=\lambda_{x}\left(e_{s}\right)$ for any $s \in \mathfrak{k}$ of unit norm, $\lambda(x ; \alpha s)=\alpha \lambda(x ; s)$ for any positive real number $\alpha$, and any point in $\partial_{\infty}(K \backslash G)$ is of the form $e_{s}$ for some $s$. Hence, the notion of analytic stability in the present paper coincides with the notion given in M]. In order to rephrase the definition of polystability in terms of the functions $\lambda(x ; s)$ we need some preparation. Two skew Hermitian endomorphisms $a, b$ of a 
complex vector space $V$ are said to be opposed if $\mathbf{i} a$ and $-\mathbf{i} b$ have the same spectrum, say $\left\{\lambda_{0}<\cdots<\lambda_{r}\right\} \subset \mathbb{R}$, and the increasing filtrations $W_{a}^{\bullet}$ and $W_{b}^{\bullet}$ defined as

$$
W_{a}^{j}=\bigoplus_{i \leq j} \operatorname{Ker}\left(\mathbf{i} a-\lambda_{i} \mathrm{Id}\right), \quad W_{b}^{j}=\bigoplus_{i \geq r-j} \operatorname{Ker}\left(\mathbf{i} b+\lambda_{i} \mathrm{Id}\right)
$$

satisfy $V=\bigoplus_{p+q=r} W_{a}^{p} \cap W_{b}^{q}$. Two elements $u, v \in \mathfrak{k}$ are said to be opposed if $u$ and $-v$ belong to the same adjoint orbit in $\mathfrak{k}$ and $\operatorname{ad}(u), \operatorname{ad}(v)$ are opposed endomorphisms of $\mathfrak{g}$. For example, for any $u \in \mathfrak{k}$ the elements $u,-u$ are opposed. Note also that if $u$ has unit norm and $-v$ belongs to the adjoint orbit through $u$, then $v$ also has unit norm because the norm in $\mathfrak{k}$ is invariant. The following lemma is a consequence of Lemma 2.3 below.

Lemma 1.4. A point $x \in X$ is polystable if $\lambda(x ; s) \geq 0$ for any $s \in \mathfrak{k}$ and if, for any nonzero $s \in \mathfrak{k}$ such that $\lambda(x ; s)=0$, there exists some $u \in \mathfrak{k}$ which is opposed to $s$ and such that $\lambda(x ; u)=0$.

The results in this paper can be seen as an analytic version of part of the results in Chapter 2 of MFK. Mumford's point of view is that the maximal weights in the case of projective varieties define a function on the flag complex $\Delta(G)$, which is the set of rational points at infinity of $G$ and can be naturally thought of as an algebraic version of the boundary $\partial_{\infty}(K \backslash G)$. More precisely, the function $\Delta(G) \ni \delta \mapsto \nu^{L}(x, \delta)$ defined on p. 59 of [op. cit.] is the analogue of our function $\lambda_{x}$. When $X$ is projective and its symplectic structure is the restriction of the Fubini-Study structure on the projective space, statement (3) in Theorem 1.3. combined with Kempf-Ness's results (see [Sch for an excellent survey), implies the usual Hilbert-Mumford criterion for stability in GIT, and this explains the title of the present paper. On the other hand, statement (4) in Theorem 1.3 gives a characterization of which points $x$ in a linear representation of a reductive group $G$ have closed orbit $G \cdot x$ uniquely in terms of maximal weights, and this seems to be a new result (note that antipodal points in $\Delta(G)$, as defined in Definition 2.8, p. 61 in [op. cit.], correspond to pairs of points in $\partial_{\infty}(K \backslash G)$ which can be connected by a geodesic).

The idea of viewing the maximal weights as defining functions on the boundary $\partial_{\infty}(K \backslash G)$ appears in a preprint of Kapovich, Leeb and Millson [KLM], applied to the particular case of flag manifolds. In their setting the maximal weight function appears as a limit of renormalized Buseman functions (technically, however, their setting is simpler because the Buseman functions are Lipschitz, as is the case in general of the integral of the moment map when $X$ is compact). They also give a characterization of polystability (to which they refer as nice semistability); see KLM, Definition 3.12].

In [T, Teleman gives a complete characterization of semistable points in terms of the maximal weights: namely, $x \in X$ is semistable iff all maximal weights are nonnegative. Of course in this result the completeness condition cannot be removed, since by definition $x$ is semistable if the closure of its orbit meets $\mu^{-1}(0)$. Nevertheless, it might be interesting to prove Teleman's characterization of semistability using the perspective adopted in the present paper (assuming, as explained, the completeness condition given in $[\mathrm{T}]$ ). Another result which might be interesting to look at from our point of view is the definition of optimal destabilizing flags given by Bruasse and Teleman in $[\mathrm{BT}$, which is an analogue of the classical result in GIT due to Kempf $[\underline{K}]$ (some results along these lines are given in Section 3.5 in $[\mathrm{KLM}]$ ). 
The rest of the paper is organized as follows. In Section 2 we recall the definition and some basic facts on the boundary at infinity of the symmetric space $K \backslash G$. In Section 3 we construct the maximal weight functions $\lambda_{x}: \partial_{\infty}(K \backslash G) \rightarrow \mathbb{R} \cup\{\infty\}$. In Section 4 we give the proof of Theorem 1.3 and, finally, in Section 5 we prove Lemma 2.3

\section{The SYMmetric SPACE $K \backslash G$ And its Boundary AT INFinity $\partial_{\infty}(K \backslash G)$}

2.1. The boundary $\partial_{\infty}(K \backslash G)$. The coset space $K \backslash G$ has a natural structure of a smooth manifold. The group $G$ acts on it by multiplication on the right: $[g] \cdot h=[g h]$ for any $g, h \in G$. Let $z_{0} \in K \backslash G$ denote the class of the identity element $1_{G} \in G$. Choose an invariant Euclidean norm on $\mathfrak{k}$. This induces a unique $G$-invariant Riemannian metric on $K \backslash G$, because the action $T(K \backslash G) \circlearrowleft G$ given by differentiating right multiplication is transitive, the stabilizer of the fiber $T_{z_{0}}(K \backslash G)$ over the identity element $1_{G} \in G$ is $K$, and the action of $K$ on $T_{z_{0}}(K \backslash G)$ can be identified with the adjoint action of $K$ on $\mathfrak{k}$ (via the natural identification $T_{z_{0}}(K \backslash G) \simeq \mathbf{i k}$ ). The geodesics corresponding to this metric are given by maps $t \mapsto\left[e^{\mathbf{i} t s} g\right] \in K \backslash G$ for any $s \in \mathfrak{k}$ and $g \in G$.

The invariant metric on $K \backslash G$ has nonpositive curvature (see [E]), so, endowed with it, $K \backslash G$ is a Hadamard space. By the general theory of Hadamard spaces (see for example $[\mathrm{B}]$ ) there is a naturally defined boundary at infinity $\partial_{\infty}(K \backslash G)$ which can be described in concrete terms using geodesic rays, i.e. maps

$$
\gamma:(0, \infty) \rightarrow K \backslash G
$$

giving a parametrization by an arc of a portion of a geodesic. Let $d$ denote the distance function between points in $K \backslash G$. Two geodesic rays $\gamma_{0}, \gamma_{1}$ are declared to be equivalent $\gamma_{0} \sim \gamma_{1}$ if the distance $d\left(\gamma_{0}(t), \gamma_{1}(t)\right)$ is bounded independently of $t$. This defines an equivalence relation and the boundary at infinity of $K \backslash G$ is the set of equivalence classes:

$$
\partial_{\infty}(K \backslash G)=\{\text { geodesic rays }\} / \sim .
$$

If $\gamma:(0, \infty) \rightarrow K \backslash G$ is a geodesic ray and $g \in G$, then we define $\gamma \cdot g$ to be the geodesic ray whose value at $t$ is $\gamma(t) \cdot g$. This defines a right action of $G$ on the set of geodesic rays. Since the action of $G$ on the right on $K \backslash G$ is by isometries, this action on the set of geodesic rays preserves the equivalence $\sim$ and hence descends to an action on $\partial_{\infty}(K \backslash G)$.

Let $S(\mathfrak{k}) \subset \mathfrak{k}$ denote the unit sphere. For any $s \in S(\mathfrak{k})$ we define $e_{s} \in \partial_{\infty}(K \backslash G)$ to be the class of the geodesic ray $\eta_{s}:(0, \infty) \rightarrow K \backslash G$ defined as $\eta_{s}(t)=\left[e^{\mathbf{i} t s}\right]$. Then the map $e: S(\mathfrak{k}) \ni s \mapsto\left[e_{s}\right] \in \partial_{\infty}(K \backslash G)$ is a bijection (see Section II.2 in [B]). We endow $\partial_{\infty}(K \backslash G)$ with the topology which makes $e$ a homeomorphism. Then the action of $G$ on $\partial_{\infty}(K \backslash G)$ is by homeomorphisms. For each $s \in S(\mathfrak{k})$ and any $g \in G$ define $s \cdot g \in S(\mathfrak{k})$ by the property that

$$
e_{s} \cdot g=e_{s \cdot g} .
$$

We remark that the boundary $\partial_{\infty}(K \backslash G)$ is independent of the chosen invariant metric on $\mathfrak{k}$. Indeed, geodesic rays do not depend on the choice of metric (they are always of the form $t \mapsto\left[e^{\text {ist }} g\right]$ ) and neither does the equivalence relation $\sim$ on geodesic rays, because the distance functions on $K \backslash G$ induced by two choices of invariant metric on $\mathfrak{k}$ are uniformly comparable. 
2.2. The case $K=\mathrm{U}(n)$ and $G=\mathrm{GL}(n, \mathbb{C})$. When $K=\mathrm{U}(n)$ (so that $G=$ $\operatorname{GL}(n, \mathbb{C}))$ the action of $G$ on $S(\mathfrak{u}(n))$ can be computed in concrete terms. Define the map $\log : G \rightarrow \mathfrak{k}$ by the condition that $\log (g)=u$ if $g=k e^{\mathrm{i} u}$ is the Cartan decomposition of $g$, so that $k \in K$ and $u \in \mathfrak{k}$. Let $s \in S(\mathfrak{u}(n))$. The matrix is is Hermitian symmetric, so it diagonalizes and has real eigenvalues, say $\lambda_{1}<$ $\cdots<\lambda_{r}$. Let $V_{j}=\operatorname{Ker}\left(\lambda_{j}-\mathbf{i} s\right)$ be the eigenspace corresponding to $\lambda_{j}$ and define $V^{k}=V_{1} \oplus \cdots \oplus V_{k}$ for any integer $k \geq 1$. Take any $g \in G$ and define

$$
V_{j}^{\infty}=\left(g^{-1}\left(V_{j-1}\right)\right)^{\perp} \cap g^{-1}\left(V_{j}\right),
$$

where $V^{\perp}$ denotes the orthogonal of $V$. Then we have a direct sum decomposition $\mathbb{C}^{n}=\bigoplus V_{j}^{\infty}$. Define $\rho_{g}(s) \in \mathfrak{u}(n)$ by the conditions that $\rho_{g}(s)$ preserves each $V_{j}^{\infty}$ and that the restriction of $\rho_{g}(s)$ to $V_{j}^{\infty}$ is given by multiplication by $-\mathbf{i} \lambda_{j}$. We claim that $\rho_{g}(s)$ is equal to $s \cdot g$. This is equivalent to the statement

$$
\rho_{g}(s)=\lim _{\tau \rightarrow \infty} \frac{1}{\tau} \log \left(e^{\mathbf{i} \tau s} g\right) .
$$

To prove (2.2) one can argue as follows. Take a very small $\epsilon>0$ (in particular, smaller than $\left.\inf \left\{\lambda_{j}-\lambda_{j-1}\right\} / 3\right)$. Using the variational description of eigenvalues and eigenspaces of $\log (h)$, one proves that for big enough $\tau$ the eigenvalues of $s_{\tau}:=\tau^{-1} \log \left(e^{\mathbf{i} \tau s} g\right)$ are contained in $\bigcup\left[\lambda_{j}-\epsilon, \lambda_{j}+\epsilon\right]$, and the number of eigenvalues in $\left[\lambda_{j}-\epsilon, \lambda_{j}+\epsilon\right]$ is equal to $\operatorname{dim} V_{j}$. Let $V_{j}^{\tau}$ be the direct sum of the eigenspaces of $s_{\tau}$ with eigenvalues contained in $\left[\lambda_{j}-\epsilon, \lambda_{j}+\epsilon\right]$. Then $V_{j}^{\tau}$ converges to $V_{j}^{\infty}$ in the Grassmannian variety.

Using the previous computations, we can also check that the map $e: S(\mathfrak{k}) \rightarrow$ $\partial_{\infty}(K \backslash G)$ is a bijection. This is equivalent to proving the existence of a bound, for each $g \in G$ and $s \in S(\mathfrak{k})$, of the form $d\left(\left[e^{\mathbf{i} t(s \cdot g)}\right],\left[e^{\mathbf{i} t s} g\right]\right) \leq C$, where the constant $C>0$ is independent of $t$. Indeed, this implies that the geodesic ray $t \mapsto\left[e^{\mathrm{i} t s} g\right]$ is equivalent to $t \mapsto\left[e^{\mathrm{i} t(s \cdot g)}\right]$, which is $e_{s \cdot g}$. The details are left as an exercise to the reader.

2.3. Tori generated by elements in $\mathfrak{k}$. For any $s \in \mathfrak{k}$ we define the torus

$$
T_{s}=\overline{\{\exp (t s) \mid t \in \mathbb{R}\}} \subset K .
$$

Lemma 2.1. For any $s \in \mathfrak{k}$ and any $g \in G$ we have $\operatorname{dim} T_{s}=\operatorname{dim} T_{s \cdot g}$.

Proof. By the Peter-Weyl theorem (see e.g. [DK, Corollary 4.6.5]) one can pick an embedding of Lie groups $K \hookrightarrow \mathrm{U}(n)$ which complexifying induces an inclusion $G \hookrightarrow \operatorname{GL}(n, \mathbb{C})$. Since the boundary at infinity does not depend on the choice of invariant metric, this inclusion induces an inclusion of boundaries $\partial_{\infty}(K \backslash G) \hookrightarrow$ $\partial_{\infty}(U(n) \backslash \mathrm{GL}(n, \mathbb{C}))$, which is equivariant with respect to the natural action of $G$ on $\partial_{\infty}(K \backslash G)$ and the action of $G$ on $\partial_{\infty}(U(n) \backslash G L(n, \mathbb{C}))$ given by the inclusion $G \hookrightarrow \operatorname{GL}(n, \mathbb{C})$ (see Section 5.1 for details). So it suffices to consider the case $K=\mathrm{U}(n)$. If $s \in \mathfrak{u}(n)$, then $\operatorname{dim} T_{s}$ is equal to the dimension of the $\mathbb{Q}$-vector space spanned by the eigenvalues of $s$. On the other hand, the observations in Section 2.2 imply that for any $s \in \mathfrak{u}(n)$ and $g \in \mathrm{GL}(n, \mathbb{C})$, the endomorphisms $s, s \cdot g \in$ End $\mathbb{C}^{n}$ have the same set of eigenvalues, so $\operatorname{dim} T_{s}=\operatorname{dim} T_{s \cdot g}$.

2.4. Geodesically connected points. Two points in $\partial_{\infty}(K \backslash G)$ are said to be geodesically connected if there is a geodesic in $K \backslash G$ which converges on one side to one of the points and on the other side to the other point. This definition is independent of the invariant metric on $\mathfrak{k}$ because the set of geodesics in $K \backslash G$ and 
the notion of convergence of rays to points in $\partial_{\infty}(K \backslash G)$ do not depend on the metric on $\mathfrak{k}$. A trivial example:

Example 2.2. For any $s \in S(\mathfrak{k})$ the points $e_{s}, e_{-s} \in \partial_{\infty}(K \backslash G)$ are geodesically connected by the geodesic $t \mapsto\left[e^{\mathrm{i} t s}\right]$.

A concrete translation into algebraic terms of the condition of being geodesically connected can be given using the notion of opposed elements in $\mathfrak{k}$ defined in the Introduction:

Lemma 2.3. Given $u, v \in S(\mathfrak{k})$, the points $e_{u}, e_{v} \in \partial_{\infty}(K \backslash G)$ are geodesically connected if and only if $u, v$ are opposed.

To avoid an excessive detour from our arguments, we postpone the proof of the lemma to Section 5 at the end of the paper.

A more synthetic characterization of geodesic connectedness may be given in terms of parabolic subgroups. We state such a translation for the sake of completeness, but we will not use it in the sequel. Recall that a parabolic subgroup of $G$ is by definition the stabilizer of a point in $\partial_{\infty}(K \backslash G)$. Two parabolic subgroups $P, P^{\prime} \subset G$ are said to be opposed if $P \cap P^{\prime}$ is a Levi subgroup (i.e., a maximal reductive subgroup) both of $P$ and $P^{\prime}$. Now, $e_{s}$ and $e_{s^{\prime}}$ are geodesically connected if and only if their stabilizers are opposed and $s,-s^{\prime}$ belong to the same coadjoint orbit in $\mathfrak{k}$.

\section{Maximal Weights as a map $\lambda_{x}: \partial_{\infty}(K \backslash G) \rightarrow \mathbb{R} \cup\{\infty\}$}

We now come back to the situation considered in the Introduction, so that $K \circlearrowright$ $X$ is a Hamiltonian action of a compact Lie group $K$ on a Kaehler manifold $X$ preserving the complex structure, and we consider the extension of this action to a holomorphic action $G \circlearrowright X$ of the complexification $G=K^{\mathbb{C}}$.

3.1. The integral of the moment map. Denote by $\pi: \mathfrak{g}=\mathfrak{k} \oplus \mathbf{i k} \rightarrow \mathfrak{i k}$ the projection to the second factor. Let $r_{g^{-1}}: G \rightarrow G$ be the map given by multiplication by $g^{-1}$ on the right, and let $D r_{g^{-1}}: T_{g} G \rightarrow T_{1_{G}} G \simeq \mathfrak{g}$ be its derivative. For any $v \in T_{g} G$ we define $v \cdot g^{-1}:=D r_{g^{-1}}(v) \in \mathfrak{g}$.

For any $x \in X$ we define a one-form $\sigma_{x} \in \Omega^{1}(G)$ as follows:

$$
\sigma_{x}(g)(v):=\left\langle\mu(g \cdot x),-\mathbf{i} \pi\left(v \cdot g^{-1}\right)\right\rangle
$$

for any $v \in T_{v} G$. It is immediate to deduce from the definition that for any $g, h \in G$ and any $v \in T_{g} G$ we have $\sigma_{x}(g h)(v \cdot h)=\sigma_{x}(g)(v)$, so that

$$
\sigma_{h x}=r_{h}^{*} \sigma_{x} .
$$

By [M, Lemma 3.1] the form $\sigma_{x}$ is exact. Hence we may define $\Psi_{x}: G \rightarrow \mathbb{R}$ to be the unique function such that $\Psi_{x}\left(1_{G}\right)=0$ and $d \Psi_{x}=\sigma_{x}$. We call $\Psi_{x}$ the integral of the moment map. Property (3.3) implies the following cocycle formula:

$$
\Psi_{x}(g)+\Psi_{g \cdot x}(h)=\Psi_{x}(h g)
$$

for any $x \in X$ and $g, h \in G$. 
3.2. Asymptotics of the integral of the moment map. Given $s \in \mathfrak{k}$ we define $\mu_{s}(x)=\langle\mu(x), s\rangle$ for any $x \in X$, and for any $t \in \mathbb{R}$ we define $\lambda_{t}(x, s)=\mu_{s}\left(e^{\mathrm{i} t s} \cdot x\right)$. Recall that for any $s \in \mathfrak{g}$ we denote by $\xi_{s} \in \mathrm{C}^{\infty}(T X)$ the vector field generated by the infinitesimal action of $s$. Since the action of $G$ on $X$ is holomorphic we have $\xi_{\text {is }}=I \xi_{s}$, where $I \in \mathcal{C}^{\infty}($ End $T X)$ is the complex structure on $X$. Denote by $\omega$ the symplectic form on $X$. Using the defining properties of the moment map we compute:

$$
\begin{aligned}
\partial_{t} \lambda_{t}(x ; s) & =\partial_{t}\left\langle\mu\left(e^{\mathrm{i} t s} \cdot x\right), s\right\rangle=\omega\left(\xi_{s}, I \xi_{s}\right)\left(e^{\mathrm{i} t s} \cdot x\right) \\
& =\left\langle\xi_{s}\left(e^{\mathrm{i} t s} \cdot x\right), \xi_{u}\left(e^{\mathrm{i} t s} \cdot x\right)\right\rangle=\left|I \xi_{s}\left(e^{\mathbf{i} t s} \cdot x\right)\right|^{2},
\end{aligned}
$$

where $\partial_{t}$ denotes the derivative with respect to $t$. This implies

$$
\lambda_{t}(x ; s)=\langle\mu(x), s\rangle+\int_{0}^{t}\left|\xi_{s}\left(e^{\mathbf{i} \tau s} \cdot x\right)\right|^{2} d \tau,
$$

and in particular $\lambda_{t}(x ; s)$ is nondecreasing as a function of $t$.

It follows from the definition of $\sigma_{x}$ that for any $s \in \mathfrak{k}$,

$$
\Psi_{x}\left(e^{\mathbf{i} t s}\right)=\int_{0}^{t} \lambda_{\tau}(x ; s) d \tau .
$$

Since $\lambda_{\tau}$ is nondecreasing we deduce that

$$
\lim _{t \rightarrow \infty} \frac{\Psi_{x}\left(e^{\mathrm{i} t s}\right)}{t}=\lambda(x ; s):=\lim _{t \rightarrow \infty} \lambda_{t}(x ; s) \in \mathbb{R} \cup\{\infty\} .
$$

The limit $\lambda(x ; s)$ is what was defined to be the maximal weight in $[\mathrm{M}$. When it is necessary to be more specific, we will say that $\lambda(x ; s)$ is the maximal weight of the action of $K$ on $X$ and we will denote it by $\lambda^{K}(x ; s)$ (this will be the case in Section 4. where different symmetry groups will be considered simultaneously).

We end this section by showing how the growth of the integral of the moment map can be used to bound the distance between points in $X$. Recall that $d_{X}$ denotes the distance function between pairs of points in $X$.

Lemma 3.1. Let $g \in G$ and $s \in \mathfrak{k}$. If $\Psi_{x}\left(e^{\mathbf{i} t s} g\right) t^{-1}$ is bounded uniformly on $t$, then $d_{X}\left(e^{\mathbf{i} t s} g \cdot x, x\right) t^{-1 / 2}$ converges to 0 as $t \rightarrow \infty$.

Proof. Using (3.4) and (3.7) we compute

$$
\lim _{t \rightarrow \infty} \frac{\Psi_{x}\left(e^{\mathrm{i} t s} g\right)}{t}=\lim _{t \rightarrow \infty} \frac{\Psi_{x}(g)+\Psi_{g \cdot x}\left(e^{\mathrm{i} t s}\right)}{t}=\lim _{t \rightarrow \infty} \frac{\Psi_{g \cdot x}\left(e^{\mathrm{i} t s}\right)}{t}=\lambda(g \cdot x ; s) .
$$

So, if $\Psi_{x}\left(e^{\mathbf{i} t s} g\right) t^{-1}$ is bounded uniformly on $t$, then, by (3.6) $), \int_{0}^{\infty}\left|\xi_{s}\left(e^{\mathbf{i} \tau s} g \cdot x\right)\right|^{2} d \tau<$ $\infty$. Since, on the other hand,

$$
d_{X}\left(e^{\mathbf{i} t s} g \cdot x, g \cdot x\right) \leq \int_{0}^{t}\left|\xi_{\mathbf{i} s}\left(e^{\mathbf{i} \tau s} g \cdot x\right)\right| d \tau=\int_{0}^{t}\left|\xi_{s}\left(e^{\mathbf{i} \tau s} g \cdot x\right)\right| d \tau,
$$

the following lemma applied to $f(\tau)=\left|\xi_{s}\left(e^{\mathbf{i} \tau s} g \cdot x\right)\right|$ implies that $d_{X}\left(e^{\mathbf{i} t s} g \cdot x\right.$, $g \cdot x) t^{-1 / 2}$ converges to 0 as $t \rightarrow \infty$. The lemma is finished by applying the triangle inequality.

Lemma 3.2. Let $f:(0, \infty) \rightarrow \mathbb{R}_{>0}$ be a nonnegative square integrable function, so that we have $\int_{0}^{\infty} f^{2}(\tau) d \tau<\infty$. Then

$$
\left(\int_{0}^{t} f(\tau) d \tau\right) t^{-1 / 2} \rightarrow 0 \quad \text { as } t \rightarrow \infty .
$$


Proof. Let $E=\int_{0}^{\infty} f^{2}(\tau) d \tau$, let $\epsilon>0$ be any real number and choose $t_{0}>0$ in such a way that $\int_{0}^{t_{0}} f(\tau)^{2} d \tau \geq(1-\epsilon) E$, so that for any $t \geq t_{0}$ we have

$$
\int_{t_{0}}^{t} f(\tau)^{2} d \tau \leq \epsilon E
$$

Then we compute, using Cauchy-Schwarz and the previous estimate:

$$
\begin{aligned}
\int_{0}^{t} f(\tau) d \tau & =\int_{0}^{t_{0}} f(\tau) d \tau+\int_{t_{0}}^{t} f(\tau) d \tau=\int_{0}^{t_{0}} f(\tau) d \tau+\int_{t_{0}}^{t}\left(\epsilon^{-1 / 2} f(\tau)\right) \epsilon^{1 / 2} d \tau \\
& \leq\left(E t_{0}\right)^{1 / 2}+\left(E \epsilon\left(t-t_{0}\right)\right)^{1 / 2}=E^{1 / 2}\left(t_{0}^{1 / 2}+\epsilon^{1 / 2}\left(t-t_{0}\right)^{1 / 2}\right) .
\end{aligned}
$$

The result follows by observing that $\left(t_{0}^{1 / 2}+\epsilon^{1 / 2}\left(t-t_{0}\right)^{1 / 2}\right) t^{-1 / 2} \rightarrow \epsilon^{1 / 2}$ as $t \rightarrow$ $\infty$.

3.3. Extending the integral of the moment map to $\partial_{\infty}(K \backslash G)$. The cocycle condition (3.4) and the fact that for any $y \in X$ the restriction of $\Psi_{y}$ to $K \subset G$ vanishes identically (which follows immediately from the definition) implies that $\Psi_{x}(k g)=\Psi_{x}(g)$ for each $k \in K$ and $g \in G$, so that $\Psi_{x}$ descends to a map

$$
\psi_{x}: K \backslash G \rightarrow \mathbb{R} .
$$

Recall that $d$ denotes the distance function between pairs of points in $K \backslash G$, and that $z_{0} \in K \backslash G$ denotes the class of the identity element in $G$. We are next going to prove that the function $\phi_{x}: K \backslash G \rightarrow \mathbb{R}$ defined as

$$
\phi_{x}(z)=\frac{\psi_{x}(z)}{d\left(z, z_{0}\right)}
$$

extends to a function on the boundary $\partial_{\infty}(K \backslash G)$. For any geodesic ray $\gamma:[0, \infty) \rightarrow$ $K \backslash G$ we define

$$
\lambda_{x}(\gamma)=\lim _{t \rightarrow \infty} \phi_{x}(\gamma(t)) \in \mathbb{R} \cup\{\infty\} .
$$

The following lemma will be used when proving that the function $\psi_{x}$ extends to the boundary $\partial_{\infty}(K \backslash G)$.

Lemma 3.3. Let $x_{0}$ be the point given by Assumption 1.2 , There exists a constant $C_{1}>0$ such that for any $x \in X$ we have $|\mu(y)| \leq C_{1}\left(1+d_{X}\left(x_{0}, x\right)^{2}\right)$.

Proof. By Assumption 1.2 there exists a constant $C>0$ such that for any $x \in X$ and any $s \in \mathfrak{k}$ we have $\left|\xi_{s}(x)\right| \leq C|s|\left(1+d_{X}\left(x_{0}, x\right)\right)$. Take any $x \in X$, let $d=d_{X}\left(x_{0}, x\right)$ and let $\alpha:[0,2 d] \rightarrow X$ be a smooth curve parameterized by the arc length and such that $\alpha(0)=x_{0}$ and $\alpha(2 d)=x$. Take any $s \in \mathfrak{k}$ and let $f(t):=\langle\mu(\alpha(t)), s\rangle$. Using the bound on $\left|\xi_{s}\right|$ and the defining properties of the moment map we compute:

$$
\begin{aligned}
f(2 d)-f(0) & =\int_{0}^{2 d} f^{\prime}(t) d t=\int_{0}^{2 d} \omega(\alpha(t))\left(\xi_{s}(\alpha(t)), \alpha^{\prime}(t)\right) d t \\
& \leq \int_{0}^{2 d}\left|\xi_{s}(\alpha(t))\right| d t \\
& \leq \int_{0}^{2 d} C|s|\left(1+d_{X}\left(x_{0}, \alpha(t)\right) d t \leq \int_{0}^{2 d} C|s|(1+t) d t\right. \\
& \leq C^{\prime}|s|\left(1+d^{2}\right),
\end{aligned}
$$

where the constant $C^{\prime}$ can be chosen independently of $x$. The result follows. 
The extendability of $\phi_{x}$ is equivalent to the fact that $\lambda_{x}(\gamma)$ only depends on the boundary point in $\partial_{\infty}(K \backslash G)$ defined by $\gamma$. This is the content of the following result.

Proposition 3.4. If the geodesic rays $\gamma_{0}, \gamma_{1}$ satisfy $\gamma_{0} \sim \gamma_{1}$, then $\lambda_{x}\left(\gamma_{0}\right)=\lambda_{x}\left(\gamma_{1}\right)$.

Proof. We may write $\gamma_{j}(t)=\left[e^{\mathrm{i} t s_{j}} g_{j}\right]$ for $j=0,1$, where $s_{j} \in \mathfrak{k}$ and $g_{j} \in G$. Assume that $\lambda_{x}\left(\gamma_{0}\right)$ is finite. Then Lemma 3.1 implies that

$$
d_{X}\left(e^{\mathrm{i} t s_{0}} g_{0} \cdot x, x\right) t^{-1 / 2} \rightarrow 0 \quad \text { as } t \rightarrow \infty .
$$

Since $\gamma_{0} \sim \gamma_{1}$, we may bound $d\left(\gamma_{0}(t), \gamma_{1}(t)\right) \leq \kappa$ uniformly on $t$. So for any $t$ we may take a smooth map $\rho_{t}:[0,1] \rightarrow G$ such that $\rho_{t}(j)=e^{\mathbf{i} s_{j} t} g_{j}$ for $j=0,1$, and such that

$$
\int_{0}^{1}\left|\rho_{t}^{\prime}(\nu) \cdot \rho_{t}(\nu)^{-1}\right| d \nu \leq \kappa
$$

the crucial thing being that $\kappa$ is independent of $t$. We now prove:

Lemma 3.5. There exists some constant $C^{\prime}$ (depending on $x$ ) such that, for any $t$ and any $\nu \in[0,1]$,

$$
d_{X}\left(e^{\mathrm{i} t s_{0}} g_{0} \cdot x, \rho_{t}(\nu) \cdot x\right) \leq C^{\prime}\left(1+d_{X}\left(x, e^{\mathbf{i} t s_{0}} g_{0} \cdot x\right)\right) .
$$

Proof. Fix some $t$ and define $c(u)=\rho_{t}(u) \cdot x$ and $r(u)=\left|\rho_{t}^{\prime}(u) \cdot \rho_{t}(u)^{-1}\right|$. Define also $f(s)=d_{X}(c(0), c(s))$. Using Assumption (1.2) we bound

$$
\begin{aligned}
\left|f^{\prime}(s)\right| & \leq\left|c^{\prime}(s)\right|=\left|\xi_{\rho_{t}^{\prime}(s) \rho_{t}(s)^{-1}}(c(s))\right| \\
& \leq C\left|\rho_{t}^{\prime}(s) \rho_{t}(s)^{-1}\right|\left(1+d_{X}\left(x_{0}, c(s)\right)\right) \\
& \leq C r(s)\left(1+d_{X}\left(x_{0}, x\right)+d_{X}(x, c(0))+d_{X}(c(0), c(s))\right) \\
& =C r(s)\left(1+d_{X}\left(x_{0}, x\right)+d_{X}(x, c(0))+f(s)\right) .
\end{aligned}
$$

So if we define $g(s)=1+d_{X}\left(x_{0}, x\right)+d_{X}(x, c(0))+f(s)$, then we have $\left|g^{\prime}(s) / g(s)\right| \leq$ $C r(s)$. Integrating over $s \in[0, \nu]$ and using (3.9) we deduce a uniform bound $g(\nu) \leq$ $C \kappa g(0)$. The result follows, since by definition $\left.d_{X}(x, c(0))=d_{X}\left(x, e^{\mathbf{i} t s_{0}} g_{0} \cdot x\right)\right)$.

Combining (3.8) with the previous lemma we deduce that for any $\nu \in[0,1]$ we have $d_{X}\left(\rho_{t}(\nu) \cdot x, x\right) t^{-1 / 2} \rightarrow 0$ as $t \rightarrow \infty$, which implies that $d_{X}\left(\rho_{t}(\nu) \cdot x\right.$, $\left.x_{0}\right) t^{-1 / 2} \rightarrow 0$ as well. Using Lemma (3.3) we get $\left|\mu\left(\rho_{t}(\nu) \cdot x\right)\right| t^{-1} \rightarrow 0$. Combining this with 3.9 we deduce

$$
\lim _{t \rightarrow \infty} \frac{\left|\Psi_{x}\left(e^{\mathbf{i} t s_{0}} g_{0}\right)-\Psi_{x}\left(e^{\mathbf{i} t s_{1}} g_{1}\right)\right|}{t}=0,
$$

because $d \Psi_{x}=\sigma_{x}$ and $\left|\sigma_{x}(g)\right| \leq|\mu(g \cdot x)|$. Hence $\lambda_{x}\left(\gamma_{0}\right)=\lambda_{x}\left(\gamma_{1}\right)$. Arguing by contradiction we deduce that if $\lambda_{x}\left(\gamma_{0}\right)=\infty$, then $\lambda_{x}\left(\gamma_{1}\right)=\infty$. For if $\lambda_{x}\left(\gamma_{1}\right)<\infty$, then, reversing the roles of $\gamma_{0}$ and $\gamma_{1}$ in the previous arguments, we get $\lambda_{x}\left(\gamma_{0}\right)=$ $\lambda_{x}\left(\gamma_{1}\right)<\infty$.

So we may define $\lambda_{x}(y):=\lambda_{x}(\gamma) \in \mathbb{R} \cup\{\infty\}$ for any $y \in \partial_{\infty}(K \backslash G)$, where $\gamma$ is any geodesic ray representing $y$. In this way we obtain a well-defined map

$$
\lambda_{x}: \partial_{\infty}(K \backslash G) \rightarrow \mathbb{R} \cup\{\infty\},
$$

which we call the maximal weight function. We now prove a crucial equivariance property of the maximal weights. 
Lemma 3.6. For any $y \in \partial_{\infty}(K \backslash G)$ and any $g \in G$ we have $\lambda_{g \cdot x}(y)=\lambda_{x}(y \cdot g)$.

Proof. Let $\gamma$ be a geodesic ray representing $y$. Using the cocycle formula (3.4) we compute

$$
\begin{aligned}
\lambda_{g \cdot x}(y) & =\lim _{t \rightarrow \infty} \phi_{g \cdot x}(\gamma(t))=\lim _{t \rightarrow \infty} \frac{\Psi_{g \cdot x}(\gamma(t))}{d\left(\gamma(t), z_{0}\right)}=\lim _{t \rightarrow \infty} \frac{\Psi_{x}(\gamma(t) g)-\Psi_{x}(g)}{d\left(\gamma(t), z_{0}\right)} \\
& =\lim _{t \rightarrow \infty} \frac{\Psi_{x}(\gamma(t) g)}{d\left(\gamma(t), z_{0}\right)}=\lim _{t \rightarrow \infty} \frac{\Psi_{x}(\gamma(t) g)}{d\left(\gamma(t) g, z_{0}\right)}=\lambda_{x}(y \cdot g),
\end{aligned}
$$

since $t \mapsto \gamma(t) g$ represents $y \cdot g$ and the quotient $d\left(\gamma(t), z_{0}\right) / d\left(\gamma(t) g, z_{0}\right)$ converges to 1 as $t \rightarrow \infty$, because $d\left(\gamma(t), z_{0}\right)=d\left(\gamma(t) g, z_{0} g\right)$ converges to $\infty$ and by the triangle inequality $\left|d\left(\gamma(t) g, z_{0} g\right)-d\left(\gamma(t) g, z_{0}\right)\right| \leq d\left(z_{0} g, z_{0}\right)$, which is independent of $t$.

3.4. Some easy properties of maximal weights. In the next two lemmas $x, x^{\prime}$ denote points in $X$ and $s, s^{\prime}$ denote elements in $\mathfrak{k}$. Recall that $\xi_{s}$ denotes the vector field on $X$ generated by the infinitesimal action of $s$.

Lemma 3.7. If $\lambda(x ; s)=\lambda(x ;-s)=0$, then $\xi_{s}(x)=0$.

Proof. By (3.6), $\lambda(x ; s)=0$ implies that $\langle\mu(x), s\rangle \leq 0$, and $\lambda(x ;-s)=0$ implies $\langle\mu(x),-s\rangle \leq 0$. Combining both inequalities we have $\langle\mu(x), s\rangle=0$. Using again (3.6) and the equality $\lambda(x ; s)=\langle\mu(x), s\rangle$ we obtain $\xi_{s}(x)=0$.

Lemma 3.8. If $\left[s, s^{\prime}\right]=0$, then $\lambda\left(x ; s+s^{\prime}\right)=\lambda(x ; s)+\lambda\left(x^{\prime} ; s\right)$.

Proof. We have

$$
\begin{aligned}
& \lambda\left(x ; s+s^{\prime}\right)=\lim _{t \rightarrow \infty}\left\langle\mu\left(e^{\mathbf{i} t\left(s+s^{\prime}\right)} \cdot x\right), s+s^{\prime}\right\rangle \\
& =\lim _{t \rightarrow \infty}\left\langle\mu\left(e^{\mathbf{i} t s} e^{\mathbf{i} t s^{\prime}} \cdot x\right), s\right\rangle+\left\langle\mu\left(e^{\mathbf{i} t s} e^{\mathbf{i} t s^{\prime}} \cdot x\right), s^{\prime}\right\rangle \text { by linearity and }\left[s, s^{\prime}\right]=0 \\
& =\lim _{t \rightarrow \infty}\left\langle\mu\left(e^{\mathbf{i} t s} \cdot x\right), s\right\rangle+\left\langle\mu\left(e^{\mathbf{i} t s^{\prime}} \cdot x\right), s^{\prime}\right\rangle \text { by equivariance of } \mu \text { and }\left[s, s^{\prime}\right]=0 \\
& =\lambda(x ; s)+\lambda\left(x ; s^{\prime}\right) .
\end{aligned}
$$

\section{Proof of Theorem 1.3}

4.1. Proofs of (1) and (2). Statement (1) follows immediately from Lemma 3.6 and the fact that the action of $G$ on $\partial_{\infty}(K \backslash G)$ extends the action by isometries on $K \backslash G$ (so that the action on $\partial_{\infty}(K \backslash G)$ of any element in $G$ sends geodesically connected points to geodesically connected points). (2) is well known, but we recall the argument for the sake of completeness. If $G \cdot x \cap \mu^{-1}(0)$ contains two different $K$-orbits, say $\mathcal{O}_{1}, \mathcal{O}_{2} \subset K$, then by Cartan's decomposition we may find points $x_{j} \in \mathcal{O}_{j}$ such that $x_{2}=e^{\mathbf{i} t s} \cdot x_{1}$. We then have $\mu_{s}\left(x_{2}\right)=\mu_{s}\left(e^{\mathbf{i} s} \cdot x\right)=\mu_{s}\left(x_{1}\right)=0$. By (3.5) we have

$$
\mu_{s}\left(e^{\mathbf{i} s} \cdot x\right)-\mu_{s}(x)=\int_{0}^{1}\left|\xi_{s}\left(e^{\mathbf{i} \tau s} \cdot x_{1}\right)\right|^{2} d \tau
$$

which implies that $\xi_{s}\left(e^{\mathbf{i} \tau s} \cdot x_{1}\right)$ vanishes for all $\tau \in[0,1]$, so that the action of $\left\{e^{\mathbf{i} t s}\right.$ $t \in \mathbb{R}\}$ fixes $x_{1}$. Consequently, $x_{2}=x_{1}$, which implies $\mathcal{O}_{1}=\mathcal{O}_{2}$, a contradiction. 
4.2. Proof of (3). Assume that $x$ is analytically stable, so that for any $s \in S(\mathfrak{k})$ we have $\lambda(x ; s)>0$. The usual argument to prove that $G \cdot x \cap \mu^{-1}(0) \neq \emptyset$ is based on the identification between the zeroes of the moment map and the critical values of the integral of the moment map $\Psi_{x}$, and the fact that analytic stability implies that $\Psi_{x}$ is proper. Instead of following this strategy, we give here a topological argument. The condition $\lambda(x ; s)>0$ implies that there is some $\tau_{s}$ such that if $t \geq \tau_{s}$, then $\left\langle\mu\left(e^{\mathbf{i} r s} \cdot x\right), s\right\rangle>0$. Since the latter function is continuous and $S(\mathfrak{k})$ is compact, we may take some $\tau$ working for any choice of $s$, namely, such that for any $t \geq \tau$ and any $s \in S(\mathfrak{k})$ we have $\left\langle\mu\left(e^{\mathfrak{i} t s} \cdot x\right), s\right\rangle>0$. Denote by $\alpha: \mathfrak{k}^{*} \simeq \mathfrak{k}$ the isomorphism given by the pairing $\langle\cdot, \cdot\rangle$. Then the image of the map $f: S(\mathfrak{k}) \rightarrow \mathfrak{k}$ given by $f(s)=\alpha \circ \mu\left(e^{\mathbf{i} \tau s} \cdot x\right)$ is contained in $\mathfrak{k} \backslash\{0\}$, and furthermore there is a homotopy between $f$ and the identity via maps from $S(\mathfrak{k})$ to $\mathfrak{k} \backslash\{0\}$. In other words, the index of $f$ around $0 \in \mathfrak{k}$ is nontrivial, and this implies that there is some $u$ inside the ball in $\mathfrak{k}$ with boundary $S(\mathfrak{k})$ such that $f(u)=0$, which is equivalent to $\mu\left(e^{\mathbf{i} \tau u} \cdot x\right)=0$. Now to prove that $G_{x}$ is finite is equivalent to proving that $G_{y}$ is finite, where $y=e^{\mathbf{i} \tau u} \cdot x$. Since $\mu(y)=0$ and $\lambda(y ; s)>0$ for any $s$, formula (3.6) implies that for any $s \in \mathfrak{k}$ the vector field $\xi_{s}$ is nonzero at $y$. Consequently the stabilizer $K_{y}$ is finite. Finally, the condition $\mu(y)=0$ implies that $G_{y}$ is the complexification of $K_{y}$ (this is proved by checking, using (3.5), that if $k e^{\mathrm{i} u}$ fixes $y$, $k \in K$ and $u \in \mathfrak{k}$, then $\xi_{u}(y)=0$ and $k \in K_{x}$; see [Sj, Proposition 1.6]). Hence, $G_{y}$ is also finite.

The converse implication in (3) is almost immediate: if $y \in G \cdot x \cap \mu^{-1}(0)$ and $G_{x}$ is finite, then $G_{y}$ is also finite. This implies that $\xi_{u}(y) \neq 0$ for any $u \in S(\mathfrak{k})$, and now (3.5) implies that $\lambda(y ; s)>0$, so $y$ is analytically stable. From (1) it now follows that $x$ is also analytically stable.

4.3. Proof of (4). We first prove that if $x$ is polystable, then $G \cdot x \cap \mu^{-1}(0) \neq \emptyset$. Since (3) has been proved, we only need to consider strictly polystable points $x$ (namely, unstable polystable points). So let $x \in X$ be such a point. Then one can choose $s \in S(\mathfrak{k})$ such that $\lambda_{x}\left(e_{s}\right)=0$ and such that $\operatorname{dim} T_{s^{\prime}} \leq \operatorname{dim} T_{s}$ for any other $s^{\prime} \in S(\mathfrak{k})$ satisfying $\lambda_{x}\left(e_{s^{\prime}}\right)=0$.

Let $y=e_{s}$. Since $x$ is polystable, there exists some $y^{\prime} \in \partial_{\infty}(K \backslash G)$ which is geodesically connected to $y$ and such that $\lambda_{x}\left(y^{\prime}\right)=0$. Let $\gamma: \mathbb{R} \rightarrow K \backslash G$ be a parameterized geodesic in $K \backslash G$ connecting $y$ and $y^{\prime}$, and assume that $\gamma(t)=e^{\mathbf{i} u t} h$ for some $u \in \mathfrak{k}$ and $h \in G$. By (1) the point $w=h \cdot x \in X$ is polystable. If we set $u=s \cdot h^{-1}$, then we have $s^{\prime} \cdot h^{-1}=-u$, since the points $e_{s \cdot h^{-1}}$ and $e_{s^{\prime} \cdot h^{-1}}$ are connected by a geodesic passing through $z_{0} \in K \backslash G$. By Lemma 3.6 we have $\lambda_{w}\left(e_{u}\right)=\lambda_{w}\left(e_{-u}\right)=0$. In other words, $\lambda(w ; u)=\lambda(w ;-u)=0$. Then Lemma 3.7 implies that $\xi_{u}(w)=0$. Hence the group $\{\exp (t s) \mid t \in \mathbb{R}\} \subset K$ fixes $w$, and by continuity this implies that $T_{u}$ fixes $w$. So, if $\boldsymbol{t}_{u}$ denotes the Lie algebra of $T_{u}$, then for any $u^{\prime} \in \mathfrak{t}_{u}$ we have $\xi_{u^{\prime}}(w)=0$.

Lemma 4.1. For any $u^{\prime} \in \mathfrak{t}_{u}$ we have $\lambda\left(w ; u^{\prime}\right)=0$.

Proof. Since $w$ is polystable we have $\lambda\left(w ; u^{\prime}\right) \geq 0$ and $\lambda\left(w ;-u^{\prime}\right) \geq 0$. Now, (3.6) together with $\xi_{u^{\prime}}(w)=0$ implies that $\lambda\left(w ; u^{\prime}\right)=\left\langle\mu(w), u^{\prime}\right\rangle=-\left\langle\mu(w),-u^{\prime}\right\rangle=$ $-\lambda\left(w ;-u^{\prime}\right)$.

Lemmas 2.1 and 3.6 imply that $u$ has the same maximality property as $s$, namely

$$
\operatorname{dim} T_{u^{\prime}} \leq \operatorname{dim} T_{u} \text { for any } u^{\prime} \in S(\mathfrak{k}) \text { satisfying } \lambda_{w}\left(e_{u^{\prime}}\right)=0 .
$$


Let $K_{u}=\{k \in K \mid \operatorname{Ad}(k)(u)=u\}$ be the centralizer of $u$. Then $T_{u} \subset K_{u}$ is obviously central. Let $K_{0}=K_{u} / T_{u}$ and let $\mathfrak{k}_{0}$ be its Lie algebra. Consider the following maps:

(1) the projection $\pi_{u}: \mathfrak{k}^{*} \rightarrow \mathfrak{k}_{u}^{*}$ induced by the inclusion $\mathfrak{k}_{u} \subset \mathfrak{k}$, and

(2) the projection $\pi_{0}: \mathfrak{k}_{u}^{*} \rightarrow \mathfrak{k}_{0}^{*}$ induced by any linear map $\mathfrak{k}_{0} \rightarrow \mathfrak{k}_{u}$ which is a section of the projection $\mathfrak{k}_{u} \rightarrow \mathfrak{k}_{u} / \mathfrak{t}_{u}=\mathfrak{k}_{0}$ ( $\pi_{0}$ is automatically a morphism of Lie algebras because $\mathfrak{t}_{u}$ is central in $\mathfrak{k}_{u}$ ).

Let $X_{u} \subset X$ be the set of points fixed by all elements of $T_{u}$. Then $X_{u}$ is a Kaehler submanifold of $X$ and the group $K_{0}$ acts on it by isometries. A moment map for this action,

$$
\mu^{K_{0}}: X_{u} \rightarrow \mathfrak{k}_{0}^{*},
$$

can be obtained by composing $\mu^{K_{0}}=\left.\pi_{0} \circ \pi_{u} \circ \mu\right|_{X_{u}}$.

We claim that $w \in X_{u}$ is stable with respect to the action of $K_{0}$. First of all we observe that for any $u^{\prime} \in \mathfrak{t}_{u}$ we have

$$
\lambda^{K_{0}}\left(w ;\left[u^{\prime}\right]\right)=\lambda^{K}\left(w ; u^{\prime}\right),
$$

where on the left hand side we consider the maximal weights of the action of $K_{0}$ on $X_{u}$, and $\left[u^{\prime}\right]$ denotes the class in $\mathfrak{k}_{0}=\mathfrak{k}_{u} / \mathfrak{t}_{u}$ represented by $u^{\prime}$, and on the right hand side we consider the weights of the action of $K$ on $X$. It follows that

$$
\lambda^{K_{0}}\left(w ;\left[u^{\prime}\right]\right) \geq 0
$$

for any $u^{\prime} \in \mathfrak{k}_{u}$. We claim that the latter inequality is strict unless $\left[u^{\prime}\right]=0$. Indeed, if $\left[u^{\prime}\right] \neq 0$ and $\lambda^{K_{0}}\left(w ;\left[u^{\prime}\right]\right)=0$, then, letting $T \subset K$ be the torus generated by $T_{u}$ and by the closure of $\left\{\exp \left(t u^{\prime}\right) \mid t \in \mathbb{R}\right\}$, we would have, by Lemma 3.8 and arguing as in the proof of Lemma 4.1. $\lambda(w ; v)=0$ for any $v \in \mathfrak{t}=\operatorname{Lie} T$. Choosing $v$ in such a way that $\{\exp (t v) \mid t \in \mathbb{R}\}$ is dense in $T$ we would furthermore have $\operatorname{dim} T_{v}>\operatorname{dim} T_{u}$, contradicting the maximality property (4.10).

Hence $w$ is stable with respect to the action of $K_{0}$ on $X_{u}$, so by (3) there exists some $h \in G_{0}$ such that $\mu^{K_{0}}(g \cdot w)=0$. This immediately implies that $\mu^{K_{u}}(g \cdot w)=0$, where $\mu^{K_{u}}=\left.\pi_{u} \circ \mu\right|_{X_{u}}$ is the moment map for the action of $K_{u}$ on $X_{u}$ (see Lemma 4.1). We now prove that we also have $\mu(g \cdot w)=0$. Let us denote for convenience $z=g \cdot w$. Then $z$ is fixed by the action of $T_{u}$. Take a decomposition

$$
\mathfrak{k}=\mathfrak{k}_{u}^{*} \oplus \bigoplus_{\alpha} \mathfrak{k}_{\alpha}
$$

in irreducible representations of $T_{u}$, so that $\mathfrak{k}_{u}$ is the trivial representation and each $\mathfrak{k}_{\alpha}$ is nontrivial. This splitting induces a splitting of the dual vector space $\mathfrak{k}^{*}$, and we let $\mu(z)=\mu_{u}(z)+\sum \mu_{\alpha}(z)$ be the corresponding decomposition of $\mu$. We clearly have $\mu_{u}(z)=\mu^{K_{u}}(z)=0$. Now, since $z$ is fixed by $T_{u}$, the equivariance of the moment map implies that each $\mu_{\alpha}(z)$ is a $T_{u}$-invariant linear map $\mathfrak{t}_{\alpha} \rightarrow \mathbb{R}$. But each $\mathfrak{t}_{\alpha}$ is a nontrivial irreducible representation of $T_{u}$, so the following lemma implies that $\mu_{\alpha}(z)=0$.

Lemma 4.2. Let $V$ be a finite dimensional vector space and let $\Gamma \circlearrowright V$ be an irreducible nontrivial linear action. Any $\Gamma$-invariant linear function $f: V \rightarrow \mathbb{R}$ vanishes identically. 
Proof. Take any nonzero $v \in V$ which is not fixed by $\Gamma$. Then the affine closure1 $\langle\Gamma \cdot v\rangle_{\text {aff }}$ of $\Gamma \cdot v$ is equal to $V$. Indeed, $\langle\Gamma \cdot v\rangle_{\text {aff }}$ is $\Gamma$-invariant and is not a point, so if it were a proper subspace of $V$, then its translate containing the origin would be a proper nonzero invariant vector subspace of $V$, contradicting the irreducibility of $\Gamma \circlearrowright V$. Since $f$ is $\Gamma$-invariant, $f$ is constant on $\Gamma \cdot v$, and by linearity the restriction of $f$ to $\langle\Gamma \cdot v\rangle_{\text {aff }}$ is also constant. Since $0 \in\langle\Gamma \cdot v\rangle_{\text {aff }}$ and $f$ is linear, we must have $f=0$.

Since $z \in G \cdot x$, we have proved that $G \cdot x \cap \mu^{-1}(0) \neq \emptyset$.

The converse statement in (4) is almost immediate. Assume that $G \cdot x \cap \mu^{-1}(0) \neq$ $\emptyset$, and let $z \in G \cdot x \cap \mu^{-1}(0)$. By statement (1) it suffices to prove that $z$ is polystable. Since $\mu(z)=0$, (3.6) implies that $\lambda(z ; s) \geq 0$ for any $s \in S(\mathfrak{k})$, and also $\lambda(z ; s)=0$ if and only if $\xi_{s}(z)=0$. The latter implies that $\lambda(z ; s)=0$ if and only if $\lambda(z ;-s)=0$. Since $e_{s}, e_{-s}$ are always geodesically connected (see Example 2.2), it follows that $z$ is polystable.

It remains to prove that the stabilizer of polystable points is reductive. Since we have proved that if $x$ is polystable, then $G \cdot x \cap \mu^{-1}(0) \neq \emptyset$, it suffices to prove that if $\mu(z)=0$, then $G_{z}$ is reductive. This follows from the well-known observation that $G_{z}$ is the complexification of the compact group $K_{z}=\{k \in K \mid k \cdot z=z\}$.

\section{Opposed ELEMENTS IN $\mathfrak{k}$ AND GEODESICALly CONNECTED POINTS IN $\partial_{\infty}(K \backslash G)$}

The main result of this section is the proof of Lemma 2.3, which will be given in Section 5.3. In Sections 5.1 and 5.2 we state and prove some preliminary lemmas. Some of these results are probably well known to experts, but we prove them in some detail for the reader's convenience. See $\S 4.12$ in [DK] for more details.

5.1. The $K$-orbits and the $G$-orbits in $\partial_{\infty}(K \backslash G)$ are the same. Recall that for any $s \in S(\mathfrak{k})$ the stabilizer of $e_{s} \in \partial_{\infty}(K \backslash G)$ is the parabolic subgroup

$$
P_{s}=\left\{g \in G \mid e^{\mathbf{i} t s} g e^{-\mathbf{i} t s} \text { stays bounded as } t \rightarrow \infty\right\} .
$$

We check that $P_{s}$ is a Lie subgroup of $G$. Consider an injective morphism of Lie groups $\iota: G \hookrightarrow \operatorname{GL}(n, \mathbb{C}$ ) extending an inclusion $K \hookrightarrow \mathrm{U}(n)$ (as given by PeterWeyl). Using Cartan's decomposition one checks that $\iota$ is proper. Let $\delta: \mathfrak{g} \rightarrow$ $\operatorname{End}\left(\mathbb{C}^{n}\right)$ be the Lie algebra morphism induced by $\iota$. Choose $s \in S(\mathfrak{k})$, let $\nu_{1}<$ $\cdots<\nu_{k}$ be the eigenvalues of the skew symmetric endomorphism $\mathbf{i} \delta(s) \in$ End $\mathbb{C}^{n}$, and let $V_{j}=\operatorname{Ker}\left(\mathbf{i} \delta(s)-\nu_{j}\right)$. Let $Q=\left\{A \in \mathrm{GL}(n, \mathbb{C}) \mid A\left(V_{j}\right) \subset \bigoplus_{j^{\prime} \leq j} V_{j^{\prime}}\right\}$. Since $\iota$ is proper one has $P_{s}=\iota^{-1}(Q)$. Since $Q$ is closed, $P_{s}$ is also closed and hence a Lie subgroup. Its Lie algebra $\mathfrak{p}_{s}$ can be computed as follows. Let $\mathfrak{g}=\bigoplus \mathfrak{g}_{\lambda}$ be the decomposition in eigenspaces of the action of the semisimple element $\mathbf{i}$ ad $(s)$ on $\mathfrak{g}$ (so each $\lambda$ is real and $\mathbf{i}$ ad $(s)$ acts on $\mathfrak{g}_{\lambda}$ as multiplication by $\lambda$ ). Then:

$$
\mathfrak{p}_{s}=\bigoplus_{\lambda \leq 0} \mathfrak{g}_{\lambda} .
$$

As previously, we denote by $z_{0} \in K \backslash G$ the class of the identity element $1_{G} \in G$.

Lemma 5.1. For any $s \in S(\mathfrak{k})$ the action of $P_{s}$ on $K \backslash G$ is transitive.

\footnotetext{
${ }^{1}$ If $X \subset V$, the affine closure $\langle X\rangle_{\text {aff }} \subset V$ is the set of finite sums $\sum \lambda_{i} x_{i}$ with $\sum \lambda_{i}=1$ and $x_{i} \in X$.
} 
Proof. Take any $s \in S(\mathfrak{k})$. Since $K \backslash G$ is connected it suffices to check that $z_{0} \cdot P_{s}$ is open and closed in $K \backslash G$. We first prove that $\mathfrak{k}+\mathfrak{p}_{s}=\mathfrak{g}$. Let $c: \mathfrak{g} \rightarrow \mathfrak{g}$ denote the conjugation map given by the identification $\mathfrak{g} \simeq \mathfrak{k} \otimes_{\mathbb{R}} \mathbb{C}$. Since $c([a, b])=[c(a), c(b)]$ for any $a, b \in \mathfrak{g}$, we have $c \circ \mathbf{i} \operatorname{ad}(s) \circ c=-\mathbf{i}$ ad $(s)$, which implies that $c$ induces isomorphisms $\mathfrak{g}_{\lambda} \simeq \mathfrak{g}_{-\lambda}$ for each $\lambda$. Since $\mathfrak{k}$ is the fixed point set of $c$, for any nonzero $\lambda$ the intersection $\mathfrak{k} \cap\left(\mathfrak{g}_{\lambda} \oplus \mathfrak{g}_{-\lambda}\right)$ is equal to the graph of $c: \mathfrak{g}_{-\lambda} \rightarrow \mathfrak{g}_{\lambda}$, so from (5.12) we get $\mathfrak{k}+\mathfrak{p}_{s}=\mathfrak{g}$. This implies that any $g \in G$ sufficiently near $1_{G}$ can be written as $g=k \cdot p$ for some $k \in K$ and $p \in P_{s}$. Hence $z_{0} \cdot P_{s}$ contains a neighborhood of $z_{0}$. Since $P_{s}$ acts on $K \backslash G$ by homeomorphisms, this implies that $z_{0} \cdot P_{s}$ contains a neighborhood of any of its points, so it is open.

Let $\alpha: P_{s} \rightarrow K \backslash G$ be the map $p \mapsto \alpha(p)=z_{0} \cdot p$. Then $\alpha$ is the restriction of the quotient map $G \rightarrow K \backslash G$, which is proper because $K$ is compact. Since $P_{s} \subset G$ is closed, it follows that $\alpha$ is also proper, so the intersection of $\alpha\left(P_{s}\right)$ with any compact subset of $K \backslash G$ is closed. Since $K \backslash G$ is locally compact, it follows that $z_{0} \cdot P_{s}=\alpha\left(P_{s}\right)$ is closed.

Lemma 5.2. If $y, y^{\prime} \in \partial_{\infty}(K \backslash G)$ satisfy $y^{\prime}=y \cdot g$ for some $g \in G$, then there exists some $k \in K$ such that $y^{\prime}=y \cdot k$.

Proof. Assume that $y^{\prime}=y \cdot g=e_{s}$ and $z=z_{0} \cdot g$. By Lemma 5.1 there exists some $p \in P_{s}$ such that $z \cdot p=z_{0}$. Hence $k:=g p$ satisfies $y^{\prime}=y \cdot k$ and $z_{0} \cdot k=z_{0}$. So $k \in K$.

The previous lemma implies that for any $s \in \mathfrak{k}$ of unit norm there is a right action of $G$ on the adjoint orbit $\mathcal{O}_{s}=\operatorname{Ad}(K) \cdot s \subset \mathfrak{k}$. Indeed, via the map $S(\mathfrak{k}) \ni u \mapsto e_{u} \in$ $\partial_{\infty}(K \backslash G)$ the action of $K$ on the boundary $\partial_{\infty}(K \backslash G)$ corresponds to the adjoint action on $S(\mathfrak{k})$. Since the $K$-orbits in $\partial_{\infty}(K \backslash G)$ are equal to the $G$-orbits, for any $s \in S(\mathfrak{k})$ we can identify $\mathcal{O}_{s}$ with one of the $G$-orbits, and since the stabilizer of $s$ is $P_{s}$, we obtain a natural identification $\mathcal{O}_{s} \simeq P_{s} \backslash G$. By (5.12), $P_{s}$ is a complex subgroup of $G$, so we deduce that the adjoint orbits in $\mathfrak{k}$ are complex homogeneous space (this is classical; see Theorem 2 in [S] for example).

We end this paragraph with an equivariance result. Let $\rho: K \rightarrow K^{\prime}$ be a morphism of compact connected Lie groups endowed with invariant metrics, and denote by the same symbol $\rho: G \rightarrow G^{\prime}$ the induced map between the complexifications. Let $z_{0} \in K \backslash G$ and $z_{0}^{\prime} \in K^{\prime} \backslash G^{\prime}$ be the classes of the identity elements. There is a unique map $r: K \backslash G \rightarrow K^{\prime} \backslash G^{\prime}$ satisfying $r\left(z_{0} \cdot g\right)=z_{0}^{\prime} \cdot \rho(g)$ for any $g \in G$, and $r$ is Lipschitz w.r.t. the Riemannian metrics on $K \backslash G$ and $K^{\prime} \backslash G^{\prime}$. If $t \mapsto \gamma(t)=\left[e^{\mathbf{i} t s} g\right] \in K \backslash G$ is a geodesic ray, then: $t \mapsto r \circ \gamma(t)$ is a geodesic ray in $K^{\prime} \backslash G^{\prime}$ unless $d \rho(s)=0$, in which case we obtain a constant map. Since $r$ is Lipschitz, given any pair of equivalent geodesic rays $\gamma_{0} \sim \gamma_{1}$ either both $r \circ \gamma_{0}$ and $r \circ \gamma_{1}$ are geodesic rays or both are constant maps. Let $\partial_{\infty}(K \backslash G)^{\text {gen }}$ be the set of boundary points corresponding to geodesic rays which are mapped to geodesic rays. Then $r$ induces a continuous map

$$
r: \partial_{\infty}(K \backslash G)^{\text {gen }} \rightarrow \partial_{\infty}\left(K^{\prime} \backslash G^{\prime}\right) .
$$

Lemma 5.3. The set $\partial_{\infty}(K \backslash G)^{\text {gen }} \subset \partial_{\infty}(K \backslash G)$ is $G$-invariant and $r$ is equivariant, so for any $y \in \partial_{\infty}(K \backslash G)^{*}$ and $g \in G$ we have $r(y \cdot g)=r(y) \cdot \rho(g)$.

5.2. The dense orbit of the action of $P_{-s}$ on $\mathcal{O}_{s}$. Let $\mathcal{O}_{s}^{*} \subset \mathcal{O}_{s}$ denote the set of elements which are opposed to $-s$. 
Lemma 5.4. The set $\mathcal{O}_{s}^{*} \subset \mathcal{O}_{s}$ is open, dense, connected and $G$-invariant.

Proof. If $s$ belongs to the center of $\mathfrak{k}$, then $\mathcal{O}_{s}=\mathcal{O}_{s}^{*}=\{s\}$, so the statement is immediate. Now assume that $s$ is not central. Consider the Hermitian metric on $\mathfrak{g}$ which extends the invariant (Euclidean) metric on $\mathfrak{k}$ and let $K^{\prime}=\mathrm{U}(\mathfrak{g})$ and $G^{\prime}=\mathrm{GL}(\mathfrak{g})$. The morphism $\rho: G \rightarrow G^{\prime}$ given by the adjoint representation satisfies $\rho(K) \subset K^{\prime}$, so we may apply to it the considerations at the end of the previous section. In particular we have a $G$-equivariant map $r: \partial_{\infty}(K \backslash G)^{\text {gen }} \rightarrow \partial_{\infty}\left(K^{\prime} \backslash G^{\prime}\right)$. The fact that $s$ is noncentral implies that $\mathcal{O}_{s} \subset \partial_{\infty}(K \backslash G)^{\text {gen }}$, and we have $r\left(\mathcal{O}_{s}\right) \subset$ $\mathcal{O}_{\operatorname{ad}(s)}$. Let $\mathfrak{g}=\bigoplus \mathfrak{g}_{\lambda}$ be the eigenspace decomposition of the action of $\mathbf{i} \operatorname{ad}(s)$ on $\mathfrak{g}$. Let $\mathcal{F}$ be the flag manifold parameterizing increasing filtrations $\left(W^{\mu}\right)_{\mu \in \mathbb{R}}$ of complex subspaces of $\mathfrak{g}$ satisfying $\operatorname{dim} W^{\mu}=\sum_{\lambda \leq \mu} \operatorname{dim} \mathfrak{g}_{\lambda}$, endowed with the right action of $G^{\prime}$ given by $\left(W^{\mu}\right) \cdot g=\left(g^{-1} W^{\mu}\right)$. Let $\mathcal{O}_{\mathrm{ad}(s)} \subset \partial_{\infty}\left(K^{\prime} \backslash G^{\prime}\right)$ be the adjoint orbit of $\operatorname{ad}(s) \in \mathfrak{k}^{\prime}$. Consider the map $\mathcal{O}_{\text {ad }(s)} \rightarrow \mathcal{F}$ which sends $u \in \mathcal{O}_{\text {ad }(s)}$ to the filtration defined by $W^{\mu}=\sum_{\lambda \leq \mu} \operatorname{Ker}(\mathbf{i} u-\lambda)$. By the results in Section 2.2 this gives a $G^{\prime}$-equivariant bijection. Since the actions of $G^{\prime}$ on $\mathcal{O}_{\text {ad }(s)}$ and $\mathcal{F}$ are holomorphic, we deduce that the bijection $\mathcal{O}_{\text {ad }(s)} \simeq \mathcal{F}$ is a biholomorphism. Finally, Lemma 5.3 (and the fact that $G$ acts transitively on $\mathcal{O}_{s}$ ) implies that the map $\phi: \mathcal{O}_{s} \rightarrow \mathcal{F}$ obtained by composing $r$ with the isomorphism $\mathcal{O}_{\mathrm{ad}(s)} \simeq \mathcal{F}$ is holomorphic.

Let $\mathcal{F}^{*}$ be the set of $\left(W^{\mu}\right)_{\mu} \in \mathcal{F}$ such that $\mathfrak{g}=\bigoplus_{\lambda}\left(W^{\lambda} \cap\left(\bigoplus_{\nu \geq \lambda} \mathfrak{g}_{\nu}\right)\right)$, where the sum runs over the spectrum of $\mathbf{i} \operatorname{ad}(s)$. Then $\mathcal{F} \backslash \mathcal{F}^{*}$ is an analytic subvariety of $\mathcal{F}$ (in fact it is Zariski closed) and we have $\mathcal{O}_{s}^{*}=\phi^{-1}\left(\mathcal{F}^{*}\right)$. Since $r: \mathcal{O}_{s} \rightarrow \mathcal{F}$ is holomorphic we deduce that $\mathcal{O}_{s} \backslash \mathcal{O}_{s}^{*}=\phi^{-1}\left(\mathcal{F} \backslash \mathcal{F}^{*}\right)$ is an analytic subvariety of $\mathcal{O}_{s}$. Since $\mathcal{O}_{s}^{*}$ is nonempty (it contains $s$, for example), $\mathcal{O}_{s} \backslash \mathcal{O}_{s}^{*}$ is not equal to $\mathcal{O}_{s}$, and since $\mathcal{O}_{s}$ is connected this implies that $\operatorname{dim}_{\mathbb{R}} \mathcal{O}_{s} \backslash \mathcal{O}_{s}^{*} \leq \operatorname{dim}_{\mathbb{R}} \mathcal{O}_{s}-2$, so $\mathcal{O}_{s}^{*}$ is connected and dense in $\mathcal{O}_{s}$. Since $\phi$ is $G$-equivariant, $\mathcal{O}_{s}^{*} \subset \mathcal{O}_{s}$ is $G$-invariant.

We next prove that $\mathcal{O}_{s}^{*}$ is an orbit of the induced action of $P_{-s} \subset G$ on $\mathcal{O}_{s}$.

Lemma 5.5. The action of $P_{-s}$ on $\mathcal{O}_{s}^{*}$ is transitive.

Proof. Let $\mathfrak{u}=\bigoplus_{\lambda>0} \mathfrak{g}_{\lambda}$ be the Lie algebra of the biggest unipotent subgroup of $P_{-s}$. Consider the map $e: \mathfrak{u} \rightarrow \mathcal{O}_{s}^{*}$ defined as $e(u)=s \cdot e^{u}$. We are going to prove that the image of $e$ is $\mathcal{O}_{s}^{*}$. Since by Lemma 5.4, $\mathcal{O}_{s}^{*}$ is connected, it suffices to check that $e(\mathfrak{u})$ is open and closed in $\mathcal{O}_{s}^{*}$. From (5.12) we deduce that $\mathfrak{g}=\mathfrak{p}_{s} \oplus \mathfrak{u}$, so any $g \in G$ sufficiently close to $1_{G}$ can be written as $g=p e^{u}$ with $p \in P_{s}$ and $u \in \mathfrak{u}$. Hence $e(\mathfrak{u})$ is open in $P_{s} \backslash G$ (use the same arguments as in the proof of Lemma [5.1). We now prove that $e(\mathfrak{u})$ is also closed. Let the map $\phi: \mathcal{O}_{s} \rightarrow \mathcal{F}$ and the subset $\mathcal{F}^{*} \subset \mathcal{F}$ be as defined in the proof of Lemma 5.4, so that $\phi\left(\mathcal{O}_{s}^{*}\right) \subset \mathcal{F}^{*}$. There is a biholomorphism $\gamma: \mathcal{F}^{*} \rightarrow \bigoplus_{\lambda<\mu} \operatorname{Hom}\left(\mathfrak{g}_{\lambda}, \mathfrak{g}_{\mu}\right)$ characterized by the property that $\gamma^{-1}$ sends $\delta=\left(\delta_{\lambda \nu}\right) \in \bigoplus_{\lambda<\mu} \operatorname{Hom}\left(\mathfrak{g}_{\lambda}, \mathfrak{g}_{\mu}\right)$ to the filtration $\left(W^{\mu}(\delta)\right)_{\mu}$ in which $W^{\mu}(\delta)=\bigoplus_{\xi \leq \mu} \operatorname{Graph}\left(\delta_{\xi}\right)$, where $\delta_{\xi}=\sum_{\mu \geq \xi} \delta_{\xi \mu}: \mathfrak{g}_{\xi} \rightarrow \bigoplus_{\mu \geq \xi} \mathfrak{g}_{\mu}$. Now, to check that $e(\mathfrak{u}) \subset \mathcal{O}_{s}^{*}$ is closed it suffices to prove, similarly to Lemma 5.1, that the map $f=\gamma \circ \phi \circ e: \mathfrak{u} \rightarrow \bigoplus_{\lambda<\mu} \operatorname{Hom}\left(\mathfrak{g}_{\lambda}, \mathfrak{g}_{\mu}\right)$ is proper. Let $0<\lambda_{1}<\cdots<\lambda_{r}$ be the positive eigenvalues of $\mathbf{i}$ ad $(s)$. For any $u=\sum u_{\lambda_{i}} \in \mathfrak{u}$ write $u_{i}:=u_{\lambda_{i}}$, and let $f(u)_{j}$ denote the component of $f(u)$ in $\operatorname{Hom}\left(\mathfrak{g}_{0}, \mathfrak{g}_{\lambda_{j}}\right)$. We deduce from the definitions that $f(u)=\exp (-\operatorname{ad}(u))-1$ for any $u \in \mathfrak{u}$. Since $\left[\mathfrak{g}_{\lambda}, \mathfrak{g}_{\mu}\right] \subset \mathfrak{g}_{\lambda+\mu}$ (Jacobi), and the decomposition $\mathfrak{g}=\bigoplus \mathfrak{g}_{\lambda}$ is finite, there exist polynomials $P_{j}$ with

$$
f(u)_{j}=\operatorname{ad}\left(u_{j}\right)_{j}+P_{j}\left(\operatorname{ad}\left(u_{1}\right), \ldots, \operatorname{ad}\left(u_{j-1}\right)\right), \quad P_{j}(0, \ldots, 0)=0,
$$


where $\operatorname{ad}\left(u_{j}\right)_{j}$ denotes the piece of $\operatorname{ad}\left(u_{j}\right) \in \operatorname{End} \mathfrak{g}$ in $\operatorname{Hom}\left(\mathfrak{g}_{0}, \mathfrak{g}_{\lambda_{j}}\right)$. Consider the Hermitian norm on $\mathfrak{g}$ induced by the invariant norm on $\mathfrak{k}$ and define, for any $\alpha \in$ End $\mathfrak{g},|\alpha|=\sup |\alpha(v)| /|v|$, where the $v$ runs over $\mathfrak{g} \backslash\{0\}$. Given any $u \in \mathfrak{u}$, we have for each $j$ :

$$
\left|\operatorname{ad}\left(u_{j}\right)_{j}\right| \geq\left|\operatorname{ad}\left(u_{j}\right)(s)\right| /|s|=\lambda_{j}\left|u_{j}\right| .
$$

Let $f(u)_{j}$ be the piece of $f(u)$ in $\operatorname{Hom}\left(\mathfrak{g}_{0}, \mathfrak{g}_{\lambda_{j}}\right)$. Then we have $f(u)_{\lambda_{j}}=\operatorname{ad}\left(u_{\lambda_{j}}\right)$, so

$$
|f(u)| \geq\left|f(u)_{\lambda_{j}}\right| \geq\left|f(u)_{\lambda_{j}}(s)\right| /|s|=\left|\left[u_{\lambda_{j}}, s\right]\right| /|s|=\lambda_{j}\left|u_{\lambda_{j}}\right| .
$$

There exist polynomials $p_{j} \in \mathbb{C}[t]$ vanishing at $t=0$ such that for each $u_{1}, \ldots, u_{j-1}$,

$$
\left|P_{j}\left(\operatorname{ad}\left(u_{1}\right), \ldots, \operatorname{ad}\left(u_{j-1}\right)\right)\right| \leq p_{j}\left(\left|u_{1}\right|+\cdots+\left|u_{j-1}\right|\right) .
$$

Since $p_{1}(0)=\cdots=p_{r}(0)=0$, there exists an $\epsilon>0$ such that the inequalities

$$
\begin{aligned}
& t_{1}<\epsilon\left(t_{1}+\cdots+t_{r}\right), \\
& t_{2}<\epsilon\left(t_{1}+\cdots+t_{r}\right)+2 \lambda_{2}^{-1} p_{2}\left(t_{1}\right), \\
& \quad \vdots \\
& t_{r}<\epsilon\left(t_{1}+\cdots+t_{r}\right)+2 \lambda_{r}^{-1} p_{r}\left(t_{1}+\cdots+t_{r-1}\right)
\end{aligned}
$$

have no simultaneous solution $\left(t_{1}, \ldots, t_{r}\right)$ satisfying $t_{j} \geq 0$ for each $j$. Let us prove that for any $u \in \mathfrak{u}$ we have $|f(u)| \geq \lambda_{1} \epsilon|u| / 2$, which clearly implies that $f$ is proper. Define $t_{j}=\left|u_{j}\right|$ for each $j$. By the choice of $\epsilon$ at least one of the previous inequalities does not hold, say the $j$-th one. Then we have (setting $p_{1}=0$ and $P_{1}=0$ when $j=1)$

$$
\left|u_{j}\right| \geq \epsilon\left(\left|u_{1}\right|+\cdots+\left|u_{r}\right|\right)+2 \lambda_{j}^{-1} p_{j}\left(\left|u_{1}\right|+\cdots+\left|u_{j-1}\right|\right),
$$

which implies, using (5.14) and the definition of $p_{j}$,

$$
\left|\operatorname{ad}\left(u_{j}\right)_{j}\right| \geq \lambda_{j}\left|u_{j}\right| \geq 2 p_{j}\left(\left|u_{1}\right|+\cdots+\left|u_{j-1}\right|\right) \geq 2\left|P_{j}\left(\operatorname{ad}\left(u_{1}\right), \ldots, \operatorname{ad}\left(u_{j-1}\right)\right)\right| .
$$

Combining this with (5.13) we obtain

$$
\begin{aligned}
|f(u)| & \geq\left|f(u)_{j}\right| \geq\left|\operatorname{ad}\left(u_{j}\right)_{j}\right| / 2 \geq \lambda_{j}\left|u_{j}\right| / 2 \\
& \geq \lambda_{j} \epsilon\left(\left|u_{1}\right|+\cdots+\left|u_{r}\right|\right) / 2 \geq \lambda_{j} \epsilon|u| / 2 \geq \lambda_{1} \epsilon|u| / 2 .
\end{aligned}
$$

This finishes the proof of the lemma.

5.3. Proof of Lemma 2.3. Assume that $e_{u}, e_{v}$ are geodesically connected, and let $\gamma: \mathbb{R} \rightarrow K \backslash G$ be a geodesic such that $\gamma(t) \rightarrow e_{u}$ when $t \rightarrow \infty$ and $\gamma(t) \rightarrow e_{v}$ when $t \rightarrow-\infty$. By Lemma 5.1 there exists some $h \in P_{u}$ such that $\gamma(0) \cdot h=z_{0}$. Since $\gamma \cdot h$ is a geodesic passing through $z_{0}$ at time 0 , it is of the form $\gamma \cdot h(t)=e^{\mathrm{i} t s}$. We have $s=u \cdot h$ and $-s=v \cdot h$, and since $h \in P_{u}$ we have $s=u$. So $-u=v \cdot h$. By Lemma 5.2 $v$ belongs to the adjoint orbit $\mathcal{O}_{-u} \subset \mathfrak{k}$. Obviously the endomorphisms $\operatorname{ad}(u), \operatorname{ad}(-u) \in$ End $\mathfrak{g}$ are opposed, and since by Lemma 5.4. $\mathcal{O}_{u}^{*} \subset \mathcal{O}_{u}$ is $G$ invariant, $\operatorname{ad}(u), \operatorname{ad}(v)$ are opposed as well. Hence, $u$ and $v$ are opposed.

Conversely, assume that $u$ and $v$ are opposed. Then $v \in \mathcal{O}_{-u}^{*}$. By Lemma 5.5 the action of $P_{u}$ on $\mathcal{O}_{-u}^{*}$ is transitive, so there exists some $h \in P_{u}$ such that $v \cdot h=-u$. Then the geodesic $\gamma(t)=\left[e^{\mathbf{i} t u} h^{-1}\right]$ satisfies $\gamma(t) \rightarrow e_{u}$ when $t \rightarrow \infty$, and $\gamma(t) \rightarrow e_{v}$ when $t \rightarrow \infty$, so $e_{u}$ and $e_{v}$ are geodesically connected. This finishes the proof of the lemma. 


\section{REFERENCES}

[B] W. Ballmann, Lectures on spaces of nonpositive curvature, With an appendix by Misha Brin DMV Seminar 25, Birkhäuser Verlag, Basel, 1995. MR1377265 (97a:53053)

[BM] S. Bochner, D. Montgomery, Groups on analytic manifolds, Ann. of Math. (2) 48 (1947), 659-669. MR0022223 (9:174f)

[BT] L. Bruasse, A. Teleman, Harder-Narasimhan filtrations and optimal destabilizing vectors in complex geometry, Ann. Inst. Fourier (Grenoble) 55 (2005), no. 3, 1017-1053. MR2149409 (2006b:32026)

[DK] Duistermaat, Kolk, Lie groups, Universitext, Springer, 1999. MR2265844 (2007j:22016)

[E] P. Eberlein, Structure of manifolds of nonpositive curvature, Global differential geometry and global analysis 1984 (Berlin, 1984), 86-153, Lecture Notes in Math. 1156, Springer, Berlin, 1985. MR824064 (87d:53080)

[GS] V. Guillemin, S. Sternberg, Geometric Quantization and Multiplicities of Group Representations, Invent. Math. 67 (1982), 515-538. MR664118 (83m:58040)

[HH] P. Heinzner, A.T. Huckleberry, Kählerian structures on symplectic reductions, Complex Analysis and Algebraic Geometry, T. Peternell, F.-O. Schreyer, eds., W. de Gruyter, 2000. MR 1760879 (2002a:32018)

[K] G. Kempf, Instability in invariant theory, Ann. of Math. (2) 108 (1978), no. 2, 299-316. MR506989 (80c:20057)

[KLM] M. Kapovich, B. Leeb, J. Millson, Convex functions on symmetric spaces, side lengths of polygons and stability inequalities for weighted configurations, arXiv:math/0311486.

[MFK] D. Mumford, J. Fogarty, F. Kirwan, Geometric Invariant Theory, 3rd edition, Erg. Math., Springer-Verlag (1994). MR.1304906 (95m:14012)

[M] I. Mundet i Riera, A Hitchin-Kobayashi correspondence for Kaehler fibrations, J. Reine Angew. Math. 528 (2000), 41-80. MR 1801657(2002b:53035)

[Sch] G. Schwarz, The topology of algebraic quotients, in Topological methods in algebraic transformation groups (New Brunswick, NJ, 1988), 135-151, Progr. Math. 80, Birkhäuser Boston (1989). MR1040861 (90m:14043)

[SL] R. Sjamaar, E. Lerman, Stratified symplectic spaces and reduction, Ann. of Math. (2) 134 (1991), no. 2, 375-422. MR1127479 (92g:58036)

[S] J.P. Serre, Représentations linéaires et espaces homogènes Kählériens des groupes de Lie compacts (d'après Armand Borel et André Weil), Séminaire Bourbaki, Vol. 2, Exp. No. 100, 447-454, Soc. Math. France, Paris, 1995. MR.1609256

[Sj] R. Sjamaar, Holomorphic slices, symplectic reduction and multiplicities of representations, Ann. of Math. (2), 141 (1995), No. 1, 87-129. MR1314032(96a:58098)

[T] A. Teleman, Symplectic stability, analytic stability in non-algebraic complex geometry, Internat. J. Math. 15 (2004), no. 2, 183-209. MR2055369 (2005b:53138)

Departament d’Àlgebra i Geometria, Facultat de Matemàtiques, Universitat de Barcelona, Gran Via de les Corts Catalanes 585, 08007 Barcelona, Spain

E-mail address: ignasi.mundet@ub.edu 